\title{
Relationships between dietary nutrients intake and lipid levels with functional MRI dorsolateral prefrontal cortex activation
}

This article was published in the following Dove Medical Press journal:

Clinical Interventions in Aging

\author{
Huijin Lau' \\ Suzana Shahar' \\ Mazlyfarina Mohamad² \\ Nor Fadilah Rajab ${ }^{3}$ \\ Hanis Mastura Yahya' \\ Normah Che Din ${ }^{4}$ \\ Hamzaini Abdul Hamid ${ }^{5}$ \\ 'Center for Healthy Aging and \\ Wellness, Faculty of Health Sciences, \\ Universiti Kebangsaan Malaysia, \\ Kuala Lumpur, Malaysia; ${ }^{2}$ Diagnostic \\ Imaging and Radiotherapy Program, \\ Faculty of Health Sciences, Universiti \\ Kebangsaan Malaysia, Kuala Lumpur, \\ Malaysia; ${ }^{3}$ Biomedical Science Program, \\ Faculty of Health Sciences, Universiti \\ Kebangsaan Malaysia, Kuala Lumpur, \\ Malaysia; ${ }^{4}$ Health Psychology Program, \\ School of Healthcare Sciences, \\ Faculty of Health Sciences, Universiti \\ Kebangsaan Malaysia, Kuala Lumpur, \\ Malaysia; ${ }^{5}$ Department of Radiology, \\ Faculty of Medicine, Universiti \\ Kebangsaan Malaysia Medical Center, \\ Kuala Lumpur, Malaysia
}

Background: Dorsolateral prefrontal cortex (DLPFC) is a key node in the cognitive control network that supports working memory. DLPFC dysfunction is related to cognitive impairment. It has been suggested that dietary components and high-density lipoprotein cholesterol (HDL-C) play a vital role in brain health and cognitive function.

Purpose: This study aimed to investigate the relationships between dietary nutrient intake and lipid levels with functional MRI (fMRI) brain activation in DLPFC among older adults with mild cognitive impairment.

Participants and methods: A total of 15 community-dwelling older adults with mild cognitive impairment, aged $\geq 60$ years, participated in this cross-sectional study at selected senior citizen clubs in Klang Valley, Malaysia. The 7-day recall Diet History Questionnaire was used to assess participants' dietary nutrient intake. Fasting blood samples were also collected for lipid profile assessment. All participants performed N-back (0- and 1-back) working memory tasks during fMRI scanning. DLPFC (Brodmann's areas 9 and 46, and inferior, middle, and superior frontal gyrus) was identified as a region of interest for analysis.

Results: Positive associations were observed between dietary intake of energy, protein, cholesterol, vitamins B6 and B12, potassium, iron, phosphorus, magnesium, and HDL-C with DLPFC activation $(P<0.05)$. Multivariate analysis showed that vitamin B6 intake, $\beta=0.505$, $t(14)=3.29, P=0.023$, and Digit Symbol score, $\beta=0.413, t(14)=2.89, P=0.045 ; R^{2}=0.748$, were positively related to DLPFC activation.

Conclusion: Increased vitamin B6 intake and cognitive processing speed were related to greater activation in the DLPFC region, which was responsible for working memory, executive function, attention, planning, and decision making. Further studies are needed to elucidate the mechanisms underlying the association.

Keywords: brain activation, fMRI, HDL-C, vitamin B6

\section{Introduction}

Dorsolateral prefrontal cortex (DLPFC) is a key node in cognitive control network that supports working memory, executive function, attention, planning, and decision making. ${ }^{1}$ DLPFC dysfunction is attributed to synaptic neuroplasticity impairment, which in turn increases the risk of neurocognitive disorders such as Alzheimer's disease (AD). ${ }^{2}$ Lower gray matter volume in left DLPFC has also been observed in the mild cognitive impaired (MCI) group as compared to healthy control subjects. ${ }^{3}$

It has been proposed that dietary nutrients play a pivotal role in brain health and cognitive functions. For instance, the dietary intake of glucose is the fuel source for the human brain. ${ }^{4}$ Other dietary components such as omega-3-fatty acid, saturated fat,
Correspondence: Suzana Shahar Center for Healthy Aging and Wellness, Faculty of Health Sciences, Universiti Kebangsaan Malaysia, Jalan Raja Muda Abdul Aziz, 50300 Kuala Lumpur, Malaysia

Tel +60 193326530

Email suzana.shahar@ukm.edu.my 
group B vitamins, antioxidant vitamins (A, C, E), and minerals (iron, calcium, zinc, magnesium) have also been identified as having an impact on brain function and cognitive abilities. ${ }^{5}$ In addition to dietary nutrient intake, lipids are also thought to be involved in the regulation of neural functions in the central nervous system (CNS) through local mechanisms that are connected to systemic lipid metabolism. ${ }^{6}$ Emerging evidence suggests that high-density lipoprotein cholesterol (HDL-C) may play a vital role in preserving cognitive function under normal or pathological conditions. ${ }^{7}$

Previous studies have reported that the dietary intake levels of vitamin A, C, E, riboflavin, niacin, folate, calcium, and iron are inadequate among Malaysian older adults, especially among those with MCI. ${ }^{8,9}$ Hyperlipidemia and lower intake of fruits and fresh fruit juices are also significantly related to increased risk of poor cognitive function in Malaysian elderly population. ${ }^{10}$ However, these relationships have never been studied using neuroimaging method among older adults with mild cognitive impairment (MCI). Neuroimaging may be a useful tool in helping to elucidate the relationships and effects of dietary intake and blood markers with cognitive function. It would be informative if we can determine the changes in the underlying brain activation associated with objective cognitive measurements. Therefore, the aim of this study was to examine the relationships between dietary nutrient intake and lipid levels with DLPFC activation using functional MRI (fMRI) among Malaysian older adults with MCI.

\section{Participants and methods}

This study has been approved by the Universiti Kebangsaan Malaysia (UKM) Ethics Committee (IEC; NN-2017-036), and written informed consent was obtained from all participants prior to testing.

\section{Participants}

A total of 15 community-dwelling older adults with MCI, aged $\geq 60$ years, participated in this study. All participants were screened for eligibility prior to the study based on inclusion and exclusion criteria.

\section{Cognitive function and dietary nutrient intake assessments}

The participants participated in a questionnaire interviewing session conducted by trained fieldworkers at selected senior citizen clubs in Klang Valley, Malaysia. The MCI status of the participants was determined based on the criteria reported in a previous study. ${ }^{11}$ A series of neurocognitive tests (Mini Mental State of Examination, Digit Span, Rey Auditory
Verbal Learning Test, Digit Symbol, and Visual Reproduction) were used to assess the global cognitive function, working and episodic memory, cognitive processing speed, and visual memory of the participants. The dietary macronutrient and micronutrient intake of the participants was also assessed using the 7-day recall Diet History Questionnaire (DHQ). ${ }^{12}$ Standardized pictures were provided to guide the participants in filling the questionnaire. They were asked to recall all the foods and beverages consumed in the past 7 days from the day the questionnaire was administered. The resulting data were then analyzed using Nutritionist Pro $^{\text {TM }}$ Diet Analysis software (Axxya Systems, Redmond, WA, USA) to calculate the nutrient intake.

\section{Lipid profile assessment}

The participants were required to fast for at least 10 hours overnight before blood sample collection. Peripheral venous blood samples were drawn by a trained phlebotomist. In all, $5 \mathrm{~mL}$ of blood per participant were collected in tubes and stored in an ice box. Lipid profile, including total cholesterol, low-density lipoprotein cholesterol, HDL-C, triglycerides, and total cholesterol to HDL-C ratio, was analyzed using an AutoAnalyzer at a certified laboratory.

\section{fMRI and N-back task}

fMRI was conducted at the Department of Radiology, Universiti Kebangsaan Malaysia Medical Center (UKMMC), by a trained radiographer. Before the scan was carried out, the purpose, procedures, and risks were explained to the participants. All the participants completed the anatomical scans lasting 3 minutes prior to the N-back task, which lasted around 9 minutes. All subjects first viewed a PowerPoint presentation that included diagrams of blocks and a brief description of study procedure prior to completing the two tasks. N-back task, which consists of two paradigms, the 0 - and 1-back condition, was created and displayed using SuperLab 5 (Cedrus Corporation, San Pedro, CA, USA). A total of four task blocks with 15 trials each was displayed in each condition. In 0-back condition, participants were required to identify whether the item appeared to be the same as the single target at the beginning of each block. During 1-back condition, participants were required to identify whether the item displayed was the same as the one preceding it. fMRI image acquisition fMRI data were acquired on a 3.0tesla magnetic resonance (MR) scanner (Magnetom, Trio; Siemens, Erlangen, Germany). High-resolution $\mathrm{T}_{1}$-weighted anatomical images were acquired for each subject (repetition time $[\mathrm{TR}]=1,900 \mathrm{~ms}$, echo time $(\mathrm{TE})=2.35 \mathrm{~ms}$, voxel dimensions $1.0 \times 1.0 \times 1.0 \mathrm{~mm}, 250 \times 250$ voxels, 176 slices, slice 
thickness $=1 \mathrm{~mm}$ ). T2*-weighted imaging data were acquired during N-back task (TR $=3,000 \mathrm{~ms}$, $\mathrm{TE}=30 \mathrm{~ms}, 3 \mathrm{~mm}$ isotropic voxels, flip angle $=90^{\circ}, 27$ slices, slice thickness $=4 \mathrm{~mm}$ ).

\section{Preprocessing and functional imaging data analysis}

Preprocessing and data analysis were performed using Statistical Parametric Mapping software (SPM12; Wellcome Department of Cognitive Neurology, London, UK) implemented in Matlab (MathWorks Inc., Natick, MA, USA). Prior to analysis, the functional images were realigned and resliced to the mean image of the series. Functional images were co-registered to the participants' mean T1-weighted image, estimated against a standardized stereotaxic space of Montreal Neurological Institute (MNI). The normalization procedure used a six-parameter affine transformation, where the parameters constituted a spatial transformation matrix. After normalization, all functional volumes went through a spatial smoothing with a $6 \mathrm{~mm}$ full-width half-maximum isotropic Gaussian kernel in order to reduce intersubject variability.

We defined DLPFC by the intersection of Brodmann's area (BA) 9 and 46 and inferior, medial and superior frontal gyrus using WFU PickAtlas library. This region was chosen as the region of interest (ROI) as it is a key node of working and episodic memory network. ${ }^{13} \mathrm{~A}$ conventional analysis based on the general linear model was used to generate brain activation in the ROIs using the clusters containing maximum $t$ value. Individual subject analysis was performed and corrected significantly $(P<0.05$, family-wise error corrected). For each subject, the maximum $t$ value in DLPFC activation was recorded.

\section{Statistical analyses}

All data were analyzed using Statistical Package for Social Sciences (SPSS) version 22. A significant value was set at $P<0.05$. Pearson's correlation was used to examine the associations between cognitive test performance, dietary nutrient intake, and plasma lipids with DLPFC activation. In addition, the relationships between independent variables (ie, cognitive test performance, dietary nutrient intake, and lipid levels) and dependent variable (DLPFC activation) were modeled using multiple linear regression.

\section{Results}

Descriptive statistics included mean age, response accuracy, and reaction time of N-back task; DLPFC activation; lipid profile; cognitive assessments; and dietary nutrient intake level, which are demonstrated in Table 1 . The mean age of the participants was $66.8 \pm 2.70$ years. The mean response
Table I Descriptive statistics (represented as mean \pm SD)

\begin{tabular}{|c|c|c|}
\hline Parameters & $\begin{array}{l}\text { Total } \\
(\mathrm{N}=15)\end{array}$ & $\begin{array}{l}\text { Normal } \\
\text { range }\end{array}$ \\
\hline Age & $66.80 \pm 2.70$ & N/A \\
\hline Response accuracy (\%) & $47.33 \pm 18.94$ & $\mathrm{~N} / \mathrm{A}$ \\
\hline Response reaction time (seconds) & $2,264.4 I \pm 523.94$ & N/A \\
\hline $\begin{array}{l}\text { DLPFC activation (maximum } \\
t \text { value) }\end{array}$ & $13.48 \pm 3.28$ & $\mathrm{~N} / \mathrm{A}$ \\
\hline Total cholesterol (mmol/L) & $5.45 \pm 0.95$ & $<5.20$ \\
\hline Triglyceride (mmol/L) & $1.42 \pm 0.64$ & $<1.70$ \\
\hline $\mathrm{HDL}-\mathrm{C}(\mathrm{mmol} / \mathrm{L})$ & $1.56 \pm 0.40$ & $>1.03$ \\
\hline LDL-C (mmol/L) & $3.24 \pm 1.04$ & $<2.58$ \\
\hline $\mathrm{TCH}: \mathrm{HDL}-\mathrm{C}$ ratio & $3.63 \pm 0.79$ & $\mathrm{~N} / \mathrm{A}$ \\
\hline MMSE & $25.20 \pm 2.04$ & $\mathrm{~N} / \mathrm{A}$ \\
\hline Digit Span forward & $9.20 \pm 1.97$ & N/A \\
\hline Digit Span backward & $3.60 \pm 1.84$ & N/A \\
\hline Digit Symbol & $35.40 \pm 11.33$ & $\mathrm{~N} / \mathrm{A}$ \\
\hline RAVLT immediate recall & $7.13 \pm 2.07$ & $\mathrm{~N} / \mathrm{A}$ \\
\hline RAVLT delayed recall & $5.87 \pm 2.95$ & $\mathrm{~N} / \mathrm{A}$ \\
\hline VR immediate recall & $29.53 \pm 7.44$ & $\mathrm{~N} / \mathrm{A}$ \\
\hline VR delayed recall & $30.33 \pm 8.44$ & N/A \\
\hline \multicolumn{3}{|l|}{ Dietary nutrient intake } \\
\hline Energy (kcal) & $\mathrm{I}, 385.7 \mathrm{I} \pm 426.58$ & $1,770-2,330$ \\
\hline Protein $(\mathrm{g})$ & $51.09 \pm 20.38$ & $50-58$ \\
\hline Carbohydrate (\%) & $46.99 \pm 2.79$ & $50-65$ \\
\hline Fat $(g)$ & $67.06 \pm 32.53$ & $49-68$ \\
\hline Total fiber (g) & $4.60 \pm 2.83$ & $20-30$ \\
\hline Vitamin A (RE) & $645.72 \pm 284.63$ & 600 \\
\hline Vitamin C (mg) & $79.29 \pm 47.58$ & 70 \\
\hline Vitamin E (mg) & $3.04 \pm 1.15$ & $7.5-10$ \\
\hline Thiamin (mg) & $0.48 \pm 0.13$ & $1.1-1.2$ \\
\hline Riboflavin (m) & $0.85 \pm 0.29$ & $1.1-1.3$ \\
\hline Niacin (mg) & $7.79 \pm 3.86$ & $14-16$ \\
\hline Vitamin B6 (mg) & $0.94 \pm 0.70$ & $1.5-1.7$ \\
\hline Folate $(\mu \mathrm{g})$ & $71.71 \pm 27.09$ & 400 \\
\hline Vitamin $\mathrm{B} / 2(\mu \mathrm{g})$ & $3.10 \pm 3.59$ & 4 \\
\hline Sodium (mg) & $2,205 .|6 \pm 553.7|$ & 2,300 \\
\hline Potassium (mg) & $1,353.05 \pm 612.98$ & 4,700 \\
\hline Calcium (mg) & $295.54 \pm|48.6|$ & $1,000-1,200$ \\
\hline Iron (mg) & $9.28 \pm 4.21$ & $11-14$ \\
\hline Phosphorus (mg) & $696.62 \pm 298.58$ & 4,000 \\
\hline Magnesium (mg) & $118.53 \pm 57.07$ & 420 \\
\hline Zinc (mg) & $2.93 \pm 1.43$ & $4.3-6.3$ \\
\hline Copper (mg) & $0.52 \pm 0.21$ & 0.9 \\
\hline Manganese (mg) & $0.86 \pm 2.25$ & 1.8 \\
\hline Selenium $(\mu g)$ & $26.34 \pm 17.03$ & $23-31$ \\
\hline Chromium $(\mu \mathrm{g})$ & $0.04 \pm 1.00$ & $20-30$ \\
\hline Molybdenum (mg) & $0.03 \pm 0.09$ & 0.045 \\
\hline
\end{tabular}

Abbreviations: DLPFC, dorsolateral prefrontal cortex; HDL-C, high-density lipoprotein cholesterol; LDL-C, low-density lipoprotein cholesterol; MMSE, MiniMental State Examination; RAVLT, Rey Auditory Verbal Learning Test; TCH:HDL-C, total cholesterol to high-density lipoprotein cholesterol; VR, visual reproduction. 
Table 2 Activated brain regions during N-back task ( $P<0.05$, corrected for FWE)

\begin{tabular}{|l|l|l|l|l|}
\hline Anatomical locations & $\mathbf{x}, \mathbf{y}, \mathbf{z}$ & Voxels activated & Maximum $t$ value & $\boldsymbol{P}$-value \\
\hline Right middle frontal gyrus & $38,6,56$ & 14,810 & 25.12 & $<0.00 I$ \\
\hline Left superior frontal gyrus & $-4,32,38$ & 60 & 11.84 & $<0.00 I$ \\
\hline Right cerebral white matter & $10,26,36$ & 91 & 10.85 & $<0.00 I$ \\
\hline Right posterior orbital gyrus & $30,34,-18$ & 10 & 6.35 & 0.003 \\
\hline Right cerebral white matter & $24,-18,58$ & 1 & 5.86 & 0.028 \\
\hline Left cerebral white matter & $-26,44,-12$ & 8 & 5.46 & 0.004 \\
\hline Right cerebral white matter & $8,-20,70$ & 2 & 5.30 & 0.019 \\
\hline Left cerebral white matter & $-18,50,-10$ & 2 & 5.29 & 0.019 \\
\hline Right superior frontal gyrus & $14,46,44$ & 7 & 5.18 & 0.006 \\
\hline Right superior frontal gyrus & $8,50,44$ & 2 & 5.04 & 0.019 \\
\hline
\end{tabular}

Abbreviation: FWE, family-wise error corrected.

accuracy and reaction time of N-back task was $47.33 \%$ and 2,264.41 \pm 523.94 seconds, respectively. The maximum activation (maximum $t$ value) in the DLPFC region was $13.48 \pm 3.28$.

Activated brain regions during N-back task $(P<0.05$, family-wise error corrected) are presented in Table 2 and Figure 1. The total number of voxels activated in the ROIs was 22,438 voxels. Greater activation was observed in right middle frontal gyrus $(38,6,56)$ with 14,810 voxels activated, $t=25.12, P<0.001$. Other activated regions were left and right superior frontal gyrus, left and right cerebral white matter, and right posterior orbital gyrus.

Table 3 shows the associations between dietary nutrient intake, plasma lipid levels, and objective cognitive measurements with DLPFC activation. Significant positive associations were observed between dietary intake of energy ( $r=0.600, P=0.018)$, protein $(r=0.657, P=0.008)$, cholesterol $(r=0.541, P=0.037)$, riboflavin $(r=0.526, P=0.044)$, niacin $(r=0.661, P=0.007)$, vitamin B6 $(r=0.687, P=0.005)$, vitamin B12 $(r=0.547, P=0.035)$, potassium $(r=0.637$, $P=0.011)$, iron $(r=0.661, P=0.007)$, phosphorus $(r=0.556$, $P=0.031)$, and magnesium $(r=0.548, P=0.034)$ with increased DLPFC activation. A higher level of plasma HDL-C was also associated with a higher maximum $t$ value $(r=0.540$, $P=0.038)$. In addition, global cognitive function $(r=0.601$, $P=0.018)$ and cognitive processing speed $(r=0.665, P=0.007)$ as measured using Mini-Mental State Examination (MMSE) and Digit Symbol test, respectively, showed a significant positive association with increased DLPFC activation. However, age, total cholesterol, triglycerides, LDL-C levels, and TCH-HDL ratio did not demonstrate any significant association with DLPFC activation $(P>0.05)$.

Further analysis using multivariate linear regression model is presented in Table 4. The findings showed positive relationships between dietary intake of vitamin B6, $\beta=0.505, \mathrm{t}(14)=3.29, P=0.023$, and Digit Symbol, $\beta=0.413$, $\mathrm{t}(14)=2.89, P=0.045$, with DLPFC activation $\left(R^{2}=0.748\right)$.

\section{Discussion}

Subjects demonstrated a greater activation in the right middle frontal gyrus and left superior frontal gyrus during N-back task in the present study. Higher order association areas in prefrontal cortex are considered to have important roles in attention, working memory, and response selection. ${ }^{14}$ Left superior and right middle frontal gyrus as well as the inferior frontal gyrus have been proposed to support executive attention. ${ }^{15}$ Involvement of middle frontal gyrus in working memory tasks such as word reading and numerical operations was also reported in previous studies. ${ }^{16,17}$ We reasoned that the intake of certain nutrients and the levels of blood cholesterol might have associations with DLPFC activation. The brain requires much more energy compared to the other human organs. Thus, the energy transfer mechanisms from food to neurons are likely related to synaptic plasticity and the control of brain function. ${ }^{4}$ The risk of MCI or dementia was increased in subjects who derived a high percentage of their energy from carbohydrates but was reduced in those who derived a high percentage of energy from fat and protein. ${ }^{18}$ The study of Roberts et al supports our finding that participants with a higher dietary intake of energy, cholesterol, and protein also have a higher brain activation in DLPFC.

Significant associations between B complex vitamins (riboflavin, niacin, vitamin B6, and B12) and minerals (potassium, phosphorus, and magnesium) with increased activation in DLPFC have also been observed in this study. Kim et al ${ }^{19}$ found that dietary intake of riboflavin, vitamin B6, and B12 was positively associated with cognitive function measured using objective neurocognitive assessments among older 

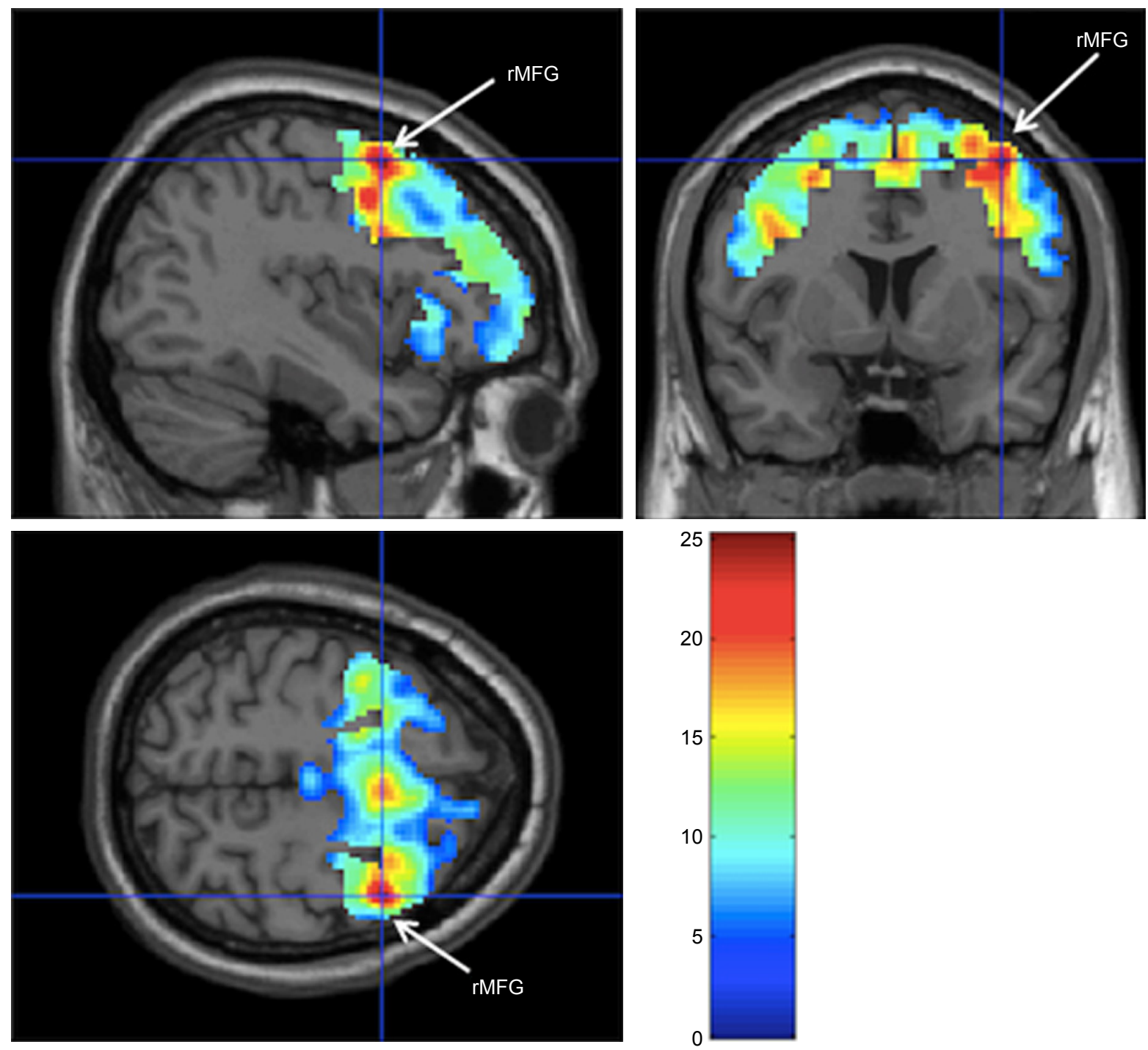

Figure I Greatest activation was observed in right middle frontal gyrus ( $x=38, y=6, z=56$ ), significant at $P<0.05$ (corrected for FWE) during N-back task among participants with mild cognitive impairment.

Abbreviations: FWE, family-wise error corrected; rMFG, right middle frontal gyrus.

adults with $\mathrm{MCI}$ and $\mathrm{AD}$. Increased intake of niacin was also significantly associated with a better performance in Digit Symbol test and negatively associated with risk of AD. ${ }^{20,21}$ Jernerén et $\mathrm{al}^{22}$ and Smith et $\mathrm{al}^{23}$ also suggested that vitamins B6 and B12 supplementations could slow down the rate of brain atrophy among older adults with MCI. Previous studies have found that high homocysteine levels have significant negative effects on cognitive function through the generation of oxidative stress, formation of $\beta$-amyloid, cerebrovascular ischemia, activation of tau kinases, and inhibition of methylation reactions. ${ }^{24} \mathrm{~A}$ higher intake of riboflavin, $\mathrm{B} 6$, and $\mathrm{B} 12$ was associated with a lower plasma homocysteine level. ${ }^{19,25,26}$ Therefore, these underlying mechanisms could explain the consistent link between B complex vitamin intake and brain function. Ozawa et $\mathrm{al}^{27}$ also suggested that, besides vitamins, a higher intake of minerals such as calcium, magnesium, and potassium was also associated with a lower risk in developing dementia.

Another main finding of the present study indicated that an increased HDL-C level was significantly associated with a greater activation in the DLPFC region. HDL-C, also known as "good cholesterol", has functions such as protection against endothelial dysfunction; inhibition of oxidative stress, anti-inflammation, and anti-thrombosis; and modulation of immune functions. ${ }^{28-31}$ High levels of HDL-C reduce the risk of cardiovascular diseases such as arteriosclerosis - a major risk factor for neurodegenerative disorders and cognitive impairment. ${ }^{7}$ There is also evidence that brain cholesterol is related to amyloid metabolism in the brain and disruption of cholesterol homeostasis in $\mathrm{AD}$ is linked to $\mathrm{A} \beta$ pathology. ${ }^{32}$ 
Table 3 Associations between dietary nutrient intake, lipid profile, cognitive assessments, and brain activation

\begin{tabular}{|c|c|c|}
\hline \multirow[t]{2}{*}{ Variables } & \multicolumn{2}{|c|}{ DLPFC activation } \\
\hline & $r$ & $P$-value ${ }^{a}$ \\
\hline \multicolumn{3}{|l|}{ Dietary nutrient intake } \\
\hline Energy (kcal) & 0.600 & $0.018^{b}$ \\
\hline Protein $(\mathrm{g})$ & 0.657 & $0.008^{c}$ \\
\hline Carbohydrate (g) & 0.335 & 0.222 \\
\hline Fat (g) & 0.421 & 0.118 \\
\hline Cholesterol (mg) & $0.54 I$ & $0.037^{\mathrm{b}}$ \\
\hline Saturated fat (g) & 0.295 & 0.285 \\
\hline Monosaturated fat (g) & 0.138 & 0.625 \\
\hline Polyunsaturated fat (g) & -0.034 & 0.905 \\
\hline Oleic acid (g) & -0.102 & 0.719 \\
\hline Linoleic acid (g) & -0.314 & 0.254 \\
\hline Linolenic acid (g) & -0.065 & 0.817 \\
\hline Total fiber $(g)$ & 0.335 & 0.223 \\
\hline Sugar (g) & 0.468 & 0.079 \\
\hline Vitamin A (retinol equivalent) & 0.148 & 0.600 \\
\hline Beta-carotene (mg) & 0.204 & 0.465 \\
\hline Vitamin C (mg) & -0.015 & 0.958 \\
\hline Vitamin E (mg) & 0.229 & $0.4 I I$ \\
\hline Thiamin (mg) & 0.358 & 0.190 \\
\hline Riboflavin (m) & 0.526 & 0.044 \\
\hline Niacin (mg) & 0.661 & $0.007^{c}$ \\
\hline Vitamin B6 (mg) & 0.687 & $0.005^{c}$ \\
\hline Folate (mg) & 0.134 & 0.635 \\
\hline Vitamin BI2 (mg) & 0.547 & $0.035^{b}$ \\
\hline Sodium (mg) & 0.480 & 0.070 \\
\hline Potassium (mg) & 0.637 & $0.01 \mathrm{I}^{\mathrm{b}}$ \\
\hline Calcium (mg) & 0.431 & 0.109 \\
\hline Iron (mg) & 0.661 & $0.007^{c}$ \\
\hline Phosphorus (mg) & 0.556 & $0.03 I^{b}$ \\
\hline Magnesium (mg) & 0.548 & $0.034^{b}$ \\
\hline Zinc (mg) & 0.335 & 0.222 \\
\hline Copper (mg) & 0.142 & 0.614 \\
\hline Manganese (mg) & $0.08 \mathrm{I}$ & 0.775 \\
\hline Selenium (mg) & 0.515 & $0.049^{b}$ \\
\hline Chromium (mg) & 0.700 & $0.004^{c}$ \\
\hline Molybdenum (mg) & 0.383 & 0.159 \\
\hline \multicolumn{3}{|l|}{ Lipid profile } \\
\hline Total cholesterol $(\mathrm{mmol} / \mathrm{L})$ & 0.350 & 0.201 \\
\hline Triglyceride (mmol/L) & -0.205 & 0.463 \\
\hline $\mathrm{HDL}-\mathrm{C}(\mathrm{mmol} / \mathrm{L})$ & 0.540 & $0.038^{b}$ \\
\hline LDL-C (mmol/L) & 0.166 & 0.554 \\
\hline TCH:HDL-C ratio & -0.302 & 0.274 \\
\hline \multicolumn{3}{|l|}{ Cognitive assessments } \\
\hline MMSE & 0.601 & $0.018^{b}$ \\
\hline Digit Span forward & 0.099 & 0.725 \\
\hline
\end{tabular}

(Continued)
Table 3 (Continued)

\begin{tabular}{|l|l|l|}
\hline \multirow{2}{*}{ Variables } & \multicolumn{2}{l|}{ DLPFC activation } \\
\cline { 2 - 3 } & $\boldsymbol{r}$ & $\boldsymbol{P}_{\text {-value }}{ }^{\mathbf{a}}$ \\
\hline Digit Span backward & $-0.05 \mathrm{I}$ & 0.856 \\
\hline Digit Symbol & 0.667 & $0.007^{\mathrm{c}}$ \\
\hline RAVLT immediate recall & 0.393 & 0.147 \\
\hline RAVLT delayed recall & 0.336 & 0.220 \\
\hline VR immediate recall & 0.440 & 0.101 \\
\hline VR delayed recall & 0.294 & 0.288 \\
\hline
\end{tabular}

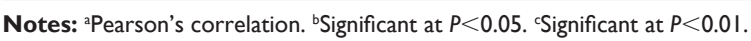
Abbreviations: DLPFC, dorsolateral prefrontal cortex; HDL-C, high-density lipoprotein cholesterol; LDL-C, low-density lipoprotein cholesterol; MMSE, MiniMental State Examination; RAVLT, Rey Auditory Verbal Learning Test; TCH:HDL-C, total cholesterol to high-density lipoprotein cholesterol; VR, visual reproduction.

HDL-C binds to $A \beta$ and maintains its solubility in cerebrospinal fluid (CSF) and plasma to prevent the formation of amyloid deposits, a pathology hallmark of AD. ${ }^{33}$ Our result is supported by several cross-sectional and longitudinal studies, which have reported that elevated HDL-C levels are positively associated with a better cognitive performance and negatively associated with the risk of MCI among elderly. ${ }^{34,35}$ Consistently, low HDL-C levels have also been found to be associated with a lower performance in working memory, immediate and delayed recall in elderly individuals. ${ }^{36} \mathrm{~A}$ previous neuroimaging study showed that young hyperlipidemic subjects have demonstrated decreased blood flow in selected brain regions. ${ }^{37}$ Higher total cholesterol has also been associated with decreased glucose metabolism in precuneus, parietotemporal, and prefrontal regions affect by $\mathrm{AD} .{ }^{38} \mathrm{~A}$ similar study that examined the association between cholesterol levels and activation intensities among midlife adults also demonstrated that a higher total cholesterol to HDL-C ratio was predictive of lower 2-back-related activation in brain regions implicated in working memory performance. ${ }^{39}$

Multivariate linear regression analysis also revealed that an increased intake of vitamin B6 was significantly related to a greater DLPFC activation. The average vitamin B6 intake of participants from the present study was $0.94 \mathrm{mg} / \mathrm{day}$, which is lower than the Malaysia recommended nutrient intakes of $1.5 \mathrm{mg} /$ day for women and $1.7 \mathrm{mg} /$ day for men. The deficiency

Table 4 Relationships between dietary nutrient intake, lipid profile, cognitive assessments, and brain activation

\begin{tabular}{|l|l|l|l|}
\hline \multirow{2}{*}{ Parameters } & \multicolumn{3}{|l|}{ DLPFC activation } \\
\cline { 2 - 4 } & OR & $\boldsymbol{t}$ & $\boldsymbol{P}_{\text {-value }}$ \\
\hline Vitamin B6 & 0.505 & 2.67 & 0.023 \\
\hline Digit Symbol & 0.413 & 2.29 & 0.045 \\
\hline
\end{tabular}

Note: ${ }^{2}$ Multiple linear regression $\left(R^{2}=0.748\right)$, significant at $P<0.05$. Abbreviation: DLPFC, dorsolateral prefrontal cortex. 
of vitamin B6 is associated with cognitive deficits and brain structural change. ${ }^{40} \mathrm{~A}$ higher intake of vitamin B6 was found to be related to a higher gray matter volume in superior frontal gyrus in healthy older adults. ${ }^{41}$ A positive correlation between intake of vitamin B6 and larger local cortical folding has also been found within inferior frontal gyrus and other parts of brain region. ${ }^{42}$ Vitamin B6 supplementation also led to a better preserved gray matter in subjects with MCI and Alzheimer's disease. ${ }^{43}$ Nevertheless, literature on the relationship between vitamin B6 intake and brain activation is less extensive as compared to cognitive function and brain structural changes. Plasma lipid levels likely mediate the relationship between nutrient intake and brain activation. An increase in vitamin B6 intake may reduce blood cholesterol. For example, in a 12-week randomized controlled trial, consumption of vitamin B6 supplement was significantly associated with a reduction in total cholesterol and HDL-C among male patients with hypertriglyceridemia. ${ }^{44}$ Vitamin B12 deficiency may also induce cholesterol biosynthesis and act as an important metabolic risk factor. ${ }^{45}$ On contrary, diet rich in saturated fats increases plasma level of total cholesterol, triglycerides, and LDL-C as compared to diet rich in monounsaturated and polyunsaturated fats. ${ }^{46}$ As mentioned earlier, the high lipid levels were associated with poor cognitive performance, reduced blood flow in brain regions, and lower brain activation. ${ }^{34-39}$

Our study also showed a positive association between MMSE score and DLPFC activation. MMSE, which measures the global cognitive function (including attention, visuospatial, and executive functions) has a high sensitivity in detecting the frontal lobe dysfunction. ${ }^{47}$ In addition, multivariate analysis also demonstrated a positive relationship between cognitive processing speed, as assessed using Digit Symbol test and DLPFC activation. DLPFC plays a pivotal role in working memory, especially in manipulation of stimuli, integration of information, selection of appropriate response during decision-making processes, and temporary storage of information. ${ }^{48,49}$ The Digit Symbol test is sensitive to cognitive changes during aging process, particularly working memory, information processing speed, and executive control function. ${ }^{50}$ This finding was supported by the evidence that cognitive processing speed is closely related to the structural integrity of white matter tracts associated with left middle frontal gyrus. ${ }^{51}$

\section{Strengths and limitations}

The strengths and limitations of the current study must be considered. The primary strength of the current study was the application of neuroimaging technique. As fMRI is noninvasive and does not involve the use of ionizing radiation, it is suitable for studies involving elderly subjects. It also provides opportunities to examine the underlying changes in cerebral hemodynamic response to modifiable lifestyle factors such as dietary intake and blood markers. Therefore, fMRI may be a more reliable and sensitive indicator of altered response among individuals at risk of cognitive impairment prior to the development of $\mathrm{AD}$ as compared to objective cognitive assessments. ${ }^{52,53}$ Nevertheless, the sample size was small, so the present findings must be considered as preliminary. Another limitation of this study is self-reported dietary intake for the past 7 days by participants with MCI may lead to inherent bias and underreporting. Nevertheless, the dietary history method has been proved to be a better dietary assessment tool as compared to a single 24-hour dietary recall in accessing intake among individual with short-term memory loss, such as MCI. ${ }^{54}$ We acknowledged that the study only focus on MCI subjects of which no comparison could be made with age-matched non-MCI subjects. Future study should consider this limitation.

The cross-sectional nature of our study is also a limitation. There is a need for prospective study to examine the relationship between dietary intake and brain activation.

\section{Conclusion}

Increased dietary nutrient intake such as energy, protein, cholesterol, vitamin B and minerals and plasma level of HDL-C were associated with greater DLPFC activation among older adults with MCI. A higher level of plasma HDL-C, better global cognitive function, and cognitive processing speed also showed positive associations with activation in DLPFC region, which are responsible for working memory, executive function, attention, planning, and decision making. By adopting a multivariate approach, increased vitamin B6 intake and cognitive processing speed were significantly related to greater DLPFC activation, with the right middle frontal gyrus demonstrating the greatest activation. Further clinical studies are needed to elucidate the mechanisms underlying these associations.

\section{Ethical approval}

All procedures performed in this study were in accordance with the ethical standards of the Universiti Kebangsaan Malaysia Medical Center institutional research committee and the 1964 Declaration of Helsinki.

\section{Acknowledgments}

The study was supported by a grant from Biotropics Sdn. Bhd, Malaysia (grant number: NN-2017-036). We acknowledge the contributions of the co-researchers, field workers, 
phlebotomists, and research and scientific officers. We also thank the participants and their family members, community leaders, and the local authorities for their cooperation throughout recruitment and data collection processes.

\section{Disclosure}

The authors report no conflicts of interest in this work.

\section{References}

1. Breukelaar IA, Antees C, Grieve SM, et al. Cognitive control network anatomy correlates with neurocognitive behavior: A longitudinal study. Hum Brain Mapp. 2017;38(2):631-643.

2. Kumar S, Zomorrodi R, Ghazala Z. Dorsolateral prefrontal cortex neuroplasticity deficits in Alzheimer's disease. JAMA. 2017;81(10):S148.

3. Pa J, Boxer A, Chao LL, et al. Clinical-neuroimaging characteristics of dysexecutive mild cognitive impairment. Ann Neurol. 2009;65(4): 414-423.

4. Vaynman S, Ying Z, Wu A, Gomez-Pinilla F. Coupling energy metabolism with a mechanism to support brain-derived neurotrophic factormediated synaptic plasticity. Neuroscience. 2006;139(4):1221-1234.

5. Gómez-Pinilla F. Brain foods: the effects of nutrients on brain function. Nat Rev Neurosci. 2008;9(7):568-578.

6. Weinstock-Guttman B, Zivadinov R, Mahfooz N, et al. Serum lipid profiles are associated with disability and MRI outcomes in multiple sclerosis. J Neuroinflammation. 2011;8:127.

7. Hottman DA, Chernick D, Cheng S, Wang Z, Li L. HDL and cognition in neurodegenerative disorders. Neurobiol Dis. 2014;72(Pt A):22-36.

8. Nik Mohd Fakhruddin NNI, Shahar S, Abd Aziz NA, Yahya HM, Rajikan R. Which aging group prone to have inadequate nutrient intake?: TUA Study. Sains Malaysiana. 2016;45(9):1381-1391.

9. Wong SH, Rajikan R, das S. Antioxidants intake and mild cognitive impairment among elderly people in Klang Valley: a pilot study. Sains Malaysiana. 2010;39(4):689-696.

10. Vanoh D, Shahar S, Din NC, et al. Predictors of poor cognitive status among older Malaysian adults: baseline findings from the LRGS TUA cohort study. Aging Clin Exp Res. 2017;29(2):173-182.

11. Shahar S, Omar A, Vanoh D, et al. Approaches in methodology for population-based longitudinal study on neuroprotective model for healthy longevity (TUA) among Malaysian Older Adults. Aging Clin Exp Res. 2016;28(6):1089-1104.

12. Shahar S, Earland J, Abdulrahman S. Validation of a dietary history questionnaire against a 7-D weighed record for estimating nutrient intake among rural elderly Malays. Malays J Nutr. 2000;6(1):33-44.

13. Preston AR, Eichenbaum H. Interplay of hippocampus and prefrontal cortex in memory. Curr Biol. 2013;23(17):R764-R773.

14. Mesulam M. Brain, mind, and the evolution of connectivity. Brain Cogn. 2000;42(1):4-6.

15. Fan J, Mccandliss BD, Fossella J, Flombaum JI, Posner MI. The activation of attentional networks. Neuroimage. 2005;26(2):471-479.

16. Fedorenko E, Duncan J, Kanwisher N. Broad domain generality in focal regions of frontal and parietal cortex. Proc Natl Acad Sci U S A. 2013;110(41):16616-16621.

17. Koyama MS, O'Connor D, Shehzad Z, Milham MP. Differential contributions of the middle frontal gyrus functional connectivity to literacy and numeracy. Sci Rep. 2017;7(1):17548.

18. Roberts RO, Roberts LA, Geda YE, et al. Relative intake of macronutrients impacts risk of mild cognitive impairment or dementia. J Alzheimers Dis. 2012;32(2):329-339.

19. Kim H, Kim G, Jang W, Kim SY, Chang N. Association between intake of $\mathrm{B}$ vitamins and cognitive function in elderly Koreans with cognitive impairment. Nutr J. 2014;13(1):118.

20. Qin B, Xun P, Jacobs DR, et al. Intake of niacin, folate, vitamin B-6, and vitamin B-12 through young adulthood and cognitive function in midlife: the Coronary Artery Risk Development in Young Adults (CARDIA) study. Am J Clin Nutr. 2017;106(4):1032-1040.
21. Morris MC, Evans DA, Bienias JL, et al. Dietary niacin and the risk of incident Alzheimer's disease and of cognitive decline. J Neurol Neurosurg Psychiatry. 2004;75(8):1093-1099.

22. Jernerén F, Elshorbagy AK, Oulhaj A, Smith SM, Refsum H, Smith AD. Brain atrophy in cognitively impaired elderly: the importance of longchain $\omega-3$ fatty acids and B vitamin status in a randomized controlled trial. Am J Clin Nutr. 2015;102(1):215-221.

23. Smith AD, Smith SM, de Jager CA, et al. Homocysteine-lowering by $\mathrm{B}$ vitamins slows the rate of accelerated brain atrophy in mild cognitive impairment: a randomized controlled trial. PLoS One. 2010; 5(9):e12244.

24. Smith AD, Refsum H. Homocysteine, B vitamins, and cognitive impairment. Annu Rev Nutr. 2016;36:211-239.

25. Clarke R, Birks J, Nexo E, et al. Low vitamin B-12 status and risk of cognitive decline in older adults. Am J Clin Nutr. 2007;86(5): 1384-1391.

26. Haan MN, Miller JW, Aiello AE, et al. Homocysteine, B vitamins, and the incidence of dementia and cognitive impairment: results from the Sacramento Area Latino Study on Aging. Am J Clin Nutr. 2007;85(2): $511-517$.

27. Ozawa M, Ninomiya T, Ohara T, et al. Self-reported dietary intake of potassium, calcium, and magnesium and risk of dementia in the Japanese: the Hisayama Study. J Am Geriatr Soc. 2012;60(8):1515-1520.

28. Mackness B, Mackness M. Anti-inflammatory properties of paraoxonase-1 in atherosclerosis. Adv Exp Med Biol. 2010;660: $143-151$.

29. Mineo C, Deguchi H, Griffin JH, Shaul PW. Endothelial and antithrombotic actions of HDL. Circ Res. 2006;98(11):1352-1364.

30. Norata GD, Pirillo A, Ammirati E, Catapano AL. Emerging role of high density lipoproteins as a player in the immune system. Atherosclerosis. 2012;220(1):11-21.

31. van den Kommer TN, Dik MG, Comijs HC, Jonker C, Deeg DJ. Role of lipoproteins and inflammation in cognitive decline: do they interact? Neurobiol Aging. 2012;33(1):196.e1-e12.

32. Eckert GP, Kirsch C, Leutz S, Wood WG, Müller WE. Cholesterol modulates amyloid beta-peptide's membrane interactions. Pharmacopsychiatry. 2003;36(Suppl 2):S136-S143.

33. Koudinov AR, Koudinova NV. Essential role for cholesterol in synaptic plasticity and neuronal degeneration. FASEB J. 2001;15(10): 1858-1860.

34. He Q, Li Q, Zhao J, et al. Relationship between plasma lipids and mild cognitive impairment in the elderly Chinese: a case-control study. Lipids Health Dis. 2016;15(1):146.

35. Lv YB, Yin ZX, Chei CL, et al. Serum cholesterol levels within the high normal range are associated with better cognitive performance among Chinese elderly. J Nutr Health Aging. 2016;20(3):280-287.

36. Ihle A, Gouveia ÉR, Gouveia BR, et al. High-density lipoprotein cholesterol level relates to working memory, immediate and delayed cued recall in Brazilian older adults: the role of cognitive reserve. Dement Geriatr Cogn Disord. 2017;44(1-2):84-91.

37. Sinha S, Misra A, Kumar V, et al. Proton magnetic resonance spectroscopy and single photon emission computed tomography study of the brain in asymptomatic young hyperlipidaemic Asian Indians in North India show early abnormalities. Clin Endocrinol. 2004;61(2): 182-189.

38. Reiman EM, Chen K, Langbaum JB, et al. Higher serum total cholesterol levels in late middle age are associated with glucose hypometabolism in brain regions affected by Alzheimer's disease and normal aging. Neuroimage. 2010;49(1):169-176.

39. Gonzales MM, Tarumi T, Eagan DE, Tanaka H, Biney FO, Haley AP. Current serum lipoprotein levels and FMRI response to working memory in midlife. Dement Geriatr Cogn Disord. 2011;31(4):259-267.

40. Selhub J, Troen A, Rosenberg IH. B vitamins and the aging brain. Nutr Rev. 2010;68(Suppl 2):S112-S118.

41. Erickson KI, Suever BL, Prakash RS, Colcombe SJ, Mcauley E, Kramer AF. Greater intake of vitamins B6 and B12 spares gray matter in healthy elderly: a voxel-based morphometry study. Brain Res. 2008; 1199:20-26. 
42. Jannusch K, Jockwitz C, Bidmon HJ, Moebus S, Amunts K, Caspers S. A complex interplay of vitamin $\mathrm{b} 1$ and $\mathrm{b} 6$ metabolism with cognition, brain structure, and functional connectivity in older adults. Front Neurosci. 2017;11:596.

43. Douaud G, Refsum H, de Jager CA, et al. Preventing Alzheimer's disease-related gray matter atrophy by B-vitamin treatment. Proc Natl Acad Sci US A. 2013;110(23):9523-9528.

44. Hlais S, Reslan DR, Sarieddine HK, et al. Effect of lysine, vitamin B(6), and carnitine supplementation on the lipid profile of male patients with hypertriglyceridemia: a 12-week, open-label, randomized, placebocontrolled trial. Clin Ther. 2012;34(8):1674-1682.

45. Adaikalakoteswari A, Finer S, Voyias PD, et al. Vitamin B12 insufficiency induces cholesterol biosynthesis by limiting s-adenosylmethionine and modulating the methylation of SREBF1 and LDLR genes. Clin Epigenetics. 2015;7(1):14.

46. Montoya MT, Porres A, Serrano S, et al. Fatty acid saturation of the diet and plasma lipid concentrations, lipoprotein particle concentrations, and cholesterol efflux capacity. Am J Clin Nutr. 2002;75(3):484-491.

47. Axelrod BN, Goldman RS, Henry RR. Sensitivity of the mini-mental state examination to frontal lobe dysfunction in normal aging. J Clin Psychol. 1992;48(1):68-71.
48. Buckholtz JW, Martin JW, Treadway MT, et al. From blame to punishment: disrupting prefrontal cortex activity reveals norm enforcement mechanisms. Neuron. 2015;87(6):1369-1380.

49. Speck O, Ernst T, Braun J, Koch C, Miller E, Chang L. Gender differences in the functional organization of the brain for working memory. Neuroreport. 2000;11(11):2581-2585.

50. Wechsler WD. Manual for the Wechsler Adult Intelligence ScaleRevised. New York: Psychological Corporation; 1981.

51. Turken A, Whitfield-Gabrieli S, Bammer R, Baldo JV, Dronkers NF, Gabrieli JD. Cognitive processing speed and the structure of white matter pathways: convergent evidence from normal variation and lesion studies. Neuroimage. 2008;42(2):1032-1044.

52. Bondi MW, Houston WS, Eyler LT, Brown GG. FMRI evidence of compensatory mechanisms in older adults at genetic risk for Alzheimer disease. Neurology. 2005;64(3):501-508.

53. Bookheimer SY, Strojwas MH, Cohen MS, et al. Patterns of brain activation in people at risk for Alzheimer's disease. $N$ Engl J Med. 2000; 343(7):450-456.

54. Hebert JR, Ockene IS, Hurley TG, Luippold R, Well AD, Harmatz MG. Development and testing of a seven-day dietary recall. J Clin Epidemiol. 1997;50(8):925-937.
Clinical Interventions in Aging

\section{Publish your work in this journal}

Clinical Interventions in Aging is an international, peer-reviewed journal focusing on evidence-based reports on the value or lack thereof of treatments intended to prevent or delay the onset of maladaptive correlates of aging in human beings. This journal is indexed on PubMed Central, MedLine,

\section{Dovepress}

CAS, Scopus and the Elsevier Bibliographic databases. The manuscript management system is completely online and includes a very quick and fair peer-review system, which is all easy to use. Visit http://www.dovepress. $\mathrm{com} /$ testimonials.php to read real quotes from published authors. 